\title{
N, P-dual doped carbon with trace Co and rich edge sites as highly efficient electrocatalyst for oxygen reduction reaction
}

\author{
Dafeng $\mathrm{Yan}^{1 \dagger}$, Lan Guo ${ }^{1 \dagger}$, Chao Xie ${ }^{1}$, Yanyong Wang ${ }^{1}$, Yunxiao $\mathrm{Li}^{1}, \mathrm{Hao}^{1}{ }^{1}$ and \\ Shuangyin Wang ${ }^{1,2,3^{*}}$
}

\begin{abstract}
Oxygen reduction reaction (ORR) is key to fuel cells and metal-air batteries which are considered as the alternative clean energy. Various carbon materials have been widely researched as ORR electrocatalysts. It has been accepted that heteroatom doping and exposure of the edge sites can effectively improve the activity of carbon materials. In this work, we used a simple method to prepare a novel N, P-dual doped carbon-based catalyst with many holes on the surface. In addition, trace level Co doping in the carbon material forming Co-N-C active species can further enhance the ORR performance. On one hand, the doping can adjust the electronic structure of carbon atoms, which would induce more active sites for ORR. And on the other hand, the holes formed on the surface of carbon nanosheets would expose more edge sites and can improve the intrinsic activity of carbon. Due to the heteroatom doping and the exposed edge sites, the prepared carbon materials showed highly excellent ORR performance, close to that of commercial $\mathrm{Pt} / \mathrm{C}$.
\end{abstract}

Keywords: electrocatalysts, oxygen reduction reaction, dualdoping, Co-N-C, edge exposed

\section{INTRODUCTION}

Fuel cells and metal-air batteries have been recognized as promising candidates for clean and efficient energy conversion devices [1-4]. Oxygen reduction reaction (ORR) in cathodic has a great impact on the commercialization of these devices for its sluggish kinetics reaction [5]. As we know, the Pt-based electrocatalysts are still the best and most used ORR catalysts. However, the Pt-based materials have high cost, $\mathrm{CO}$ poisoning and poor stability [6]. To overcome these issues, it is desirable to develop non-precious metal or metal-free electrocatalysts for ORR. Among them, carbon-based materials draw much attention due to their high surface areas, good stability and comparable activity [7-10].

Since Dai's group first reported that vertically aligned nitrogen-containing carbon nanotubes have very good ORR performance in 2009 [2], many researchers have developed various carbon materials with high ORR performance [11-18]. Many publications have shown that the heteroatom doping can adjust the electronic structure of the carbon atom, which would have a great effect on their electrochemical properties [19-22]. And then, some groups found that the doping dual heteroatoms can further improve their activity for their synergetic effect $[23,24]$. For example, Wang and co-workers reported that $\mathrm{B}, \mathrm{N}$-co-doped carbon nanotubes showed very good ORR activity. And they also prepared N, S-co-doped graphene by pyrolysis, which has much better ORR performance [25]. Recently, Wang's group firstly proved that the edges of carbon were more active for ORR than basal plane by a micro-electrochemical testing system [26]. The charge distribution of the carbon in edge sites and basal plane was much different. It can be seen obviously that the edge carbon is highly charged compared to the basal plane. Their micro-electrochemical system can provide direct evidence to prove that the activity of edge sites was higher

\footnotetext{
${ }^{1}$ State Key Laboratory of Chem/Bio-Sensing and Chemometrics, Provincial Hunan Key Laboratory for Graphene Materials and Devices, College of Chemistry and Chemical Engineering, Hunan University, Changsha 410082, China

${ }^{2}$ Key Laboratory of Optoelectronic Devices and Systems of Ministry of Education and Guangdong Province, Shenzhen University, Shenzhen 518060, China

${ }^{3}$ Shenzhen Research Institute of Hunan University, Shenzhen 518057, China

The authors contributed equally to this work.

* Corresponding author (email: shuangyinwang@hnu.edu.cn)
} 
than that of the basal plane. Guided by this finding, many researchers try to induce more holes to expose more edge sites or different kinds of defects through various methods [27-30]. Though metal-free carbon based catalysts have been widely researched, their activities still cannot fully satisfy the demand for commercialization. In addition, further studies showed that introducing transitional metal ( $\mathrm{Fe}, \mathrm{Co}$ ) to doped carbon materials forming $\mathrm{M}-\mathrm{N}-$ C could obviously improve the ORR performance [3133]. Therefore, it would be very ideal to design dualdoped $\mathrm{M}-\mathrm{N}-\mathrm{C}$ materials with many holes on their surface to expose more edge sites as ORR electrocatalysts.

Herein, we developed an efficient method for the scalable preparion of $\mathrm{N}, \mathrm{P}$ dual-doped carbon material with trace level Co doping and rich edge sites exposed. The precursor was prepared by self-assembling melamine and phytic acid to melamine-phytic acid supermolecular aggregate (MPSA) in the presence of $\mathrm{Co}^{2+}$, followed by pyrolysis under $\mathrm{Ar} / \mathrm{H}_{2}$. And then the sample was washed by acid to remove the metal Co to form the hole on the surface. The final product had graphene-like structure with only trace Co doping and rich edge sites exposed. The resulting catalyst showed very good ORR performance under alkaline condition, which was comparable to that of commerical Pt/C. This method open up an avenue to develop carbon-based electrocatalysts with high electrochemical activity.

\section{EXPERIMENTAL SECTION}

In a typical experiment of catalyst $2 \mathrm{mmol} \mathrm{Co}\left(\mathrm{NO}_{3}\right)_{2}$ powder was added into $5 \mathrm{mmol}$ phytic acid under stirring for about one hour to form a red solution. Then the above liquid was added to an aqueous solution of melamine $(10 \mathrm{mmol}$ melamine powder in $400 \mathrm{~mL}$ water) under stirring to form pink-white precipitate (MPSA-Co). The MPSA was prepared at the same condition without adding $\mathrm{Co}^{2+}$. The resultant pink-white precipitate was collected through filtration, thoroughly washed with DI water, and then dried at $80^{\circ} \mathrm{C}$ over night. Then the sample was annealed at $900^{\circ} \mathrm{C}$ for $2 \mathrm{~h}$ under $\mathrm{Ar} / \mathrm{H}_{2}$ (9:1) atmosphere (MPSA-Co-900). After that, the product was further washed in $0.5 \mathrm{~mol} \mathrm{~L}^{-1} \mathrm{H}_{2} \mathrm{SO}_{4}$ for several hours to remove the soluble metal Co (MPSA-Co-900-Acid). The morphology was investigated by scanning electron microscopy (SEM, Hitachi, S-4800) and transmission electron microscopy (TEM, Tecnai G2 F20). The Raman spectra were recorded at room temperature on a Horiba HR 800 with an argon ion laser operating at $632 \mathrm{~nm}$. The $\mathrm{X}$-ray photoelectron spectroscopy (XPS) analysis was performed on an ESCALAB 250Xi X-ray photoelectron spectrometer using $\mathrm{Mg}$ as the excitation source. The electrochemical performance of the samples were measured on an electrochemical workstation $(\mathrm{CHI} 760 \mathrm{E}, \mathrm{CH}$ Instrument) and a rotating ring disk electrode apparatus (RRDE-3A, ALS) in a three-electrode cell by using a platinum wire as counter electrode and saturated calomel electrode (SCE) as reference electrode. The working electrode was a rotating ring/disk electrode with glass carbon disk (4 $\mathrm{mm}$ in diameter). The electrolyte was bubbled with $\mathrm{O}_{2}$ or $\mathrm{N}_{2}$ for at least 30 min before each experiment.

\section{RESULTS AND DISCUSSION}

As shown in Scheme 1, the phytic acid was first coordinated with a certain amount of $\mathrm{Co}^{2+}$. Then the complex was dropped into melamine solution at room temperature under stirring. The white-pink supermolecular aggregate (MPSA-Co) was formed via a simple cooperative assembly in water after stirring for several minutes. The dried sample was then annealed at $900^{\circ} \mathrm{C}$ for $2 \mathrm{~h}$ under $\mathrm{Ar} / \mathrm{H}_{2}$ (9:1) atmosphere (MPSA-Co-900), followed by washing in $\mathrm{H}_{2} \mathrm{SO}_{4}$ to remove the metal Co and make holes on its surface (MPSA-Co-900-Acid). As shown in Fig. S1-2, both the precursor MPSA and MPSA-Co have a graphene-like layer structure, which consist of melamine-phytic acid and melamine-phytic acid-Co ${ }^{2+}$. After annealing, MPSA-Co-900 still kept its layer structure (Fig. 1a, b). The wrinkled nanosheets were interconnected each other to form a foam-like structure, which would be beneficial for the charge or proton transfer. As we annealed the sample under $\mathrm{Ar} / \mathrm{H}_{2}$, the nanoparticle was kept in the form of metal. Therefore, after washing with acid to remove the Co metal, many holes were left on the nanosheets. Fig. S3 further proved its layer structure of MPSA-900 and no nanoparticles formed on its surface. With adding the $\mathrm{Co}^{2+}$ to the precursor, Co nanoparticles formed on the surface of the nanosheets. TEM images (Fig. 2) show many holes on the

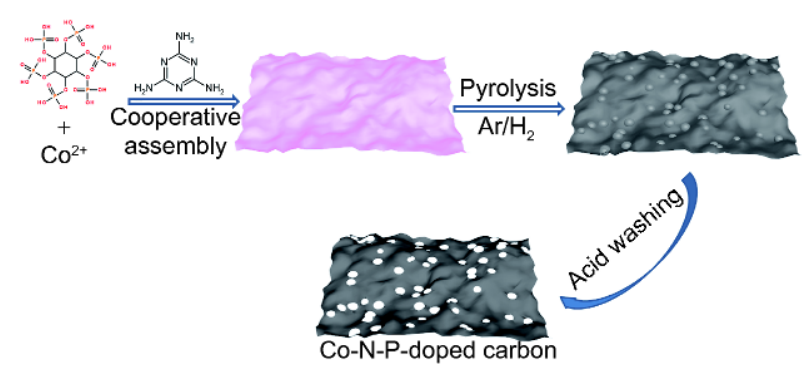

Scheme 1 The preparation process of MPSA-Co-900-Acid via cooperative assembly, pyrolysis and acid washing. 


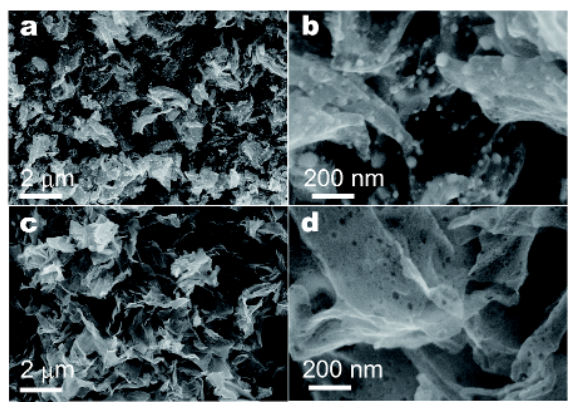

Figure 1 (a, b) SEM images of MPSA-Co-900 and (c, d) SEM images of MPSA-Co-900-Acid.
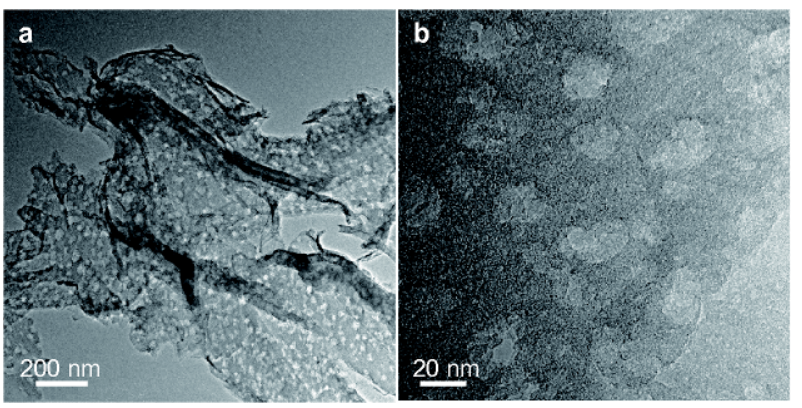

Figure 2 (a, b) TEM images of MPSA-Co-900-Acid.

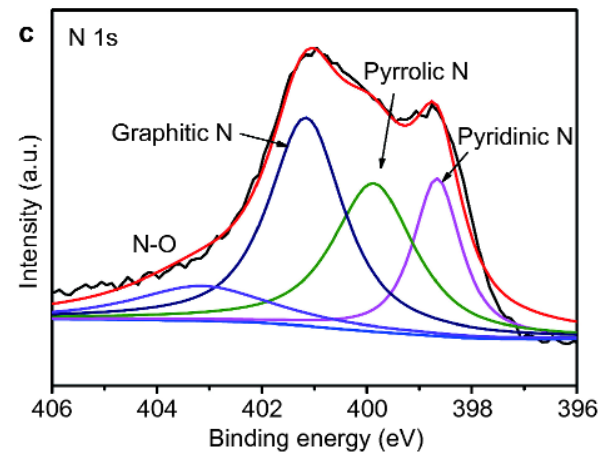

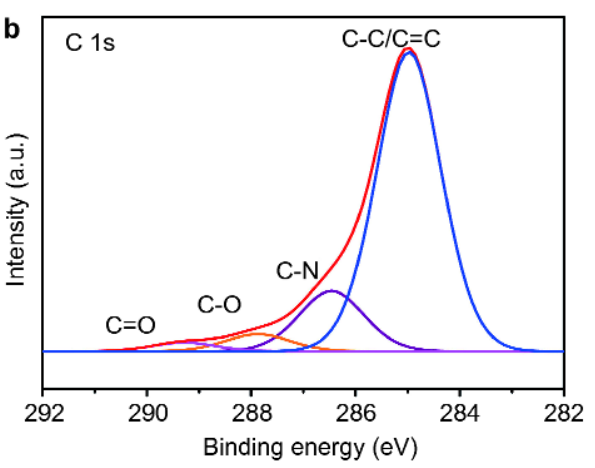
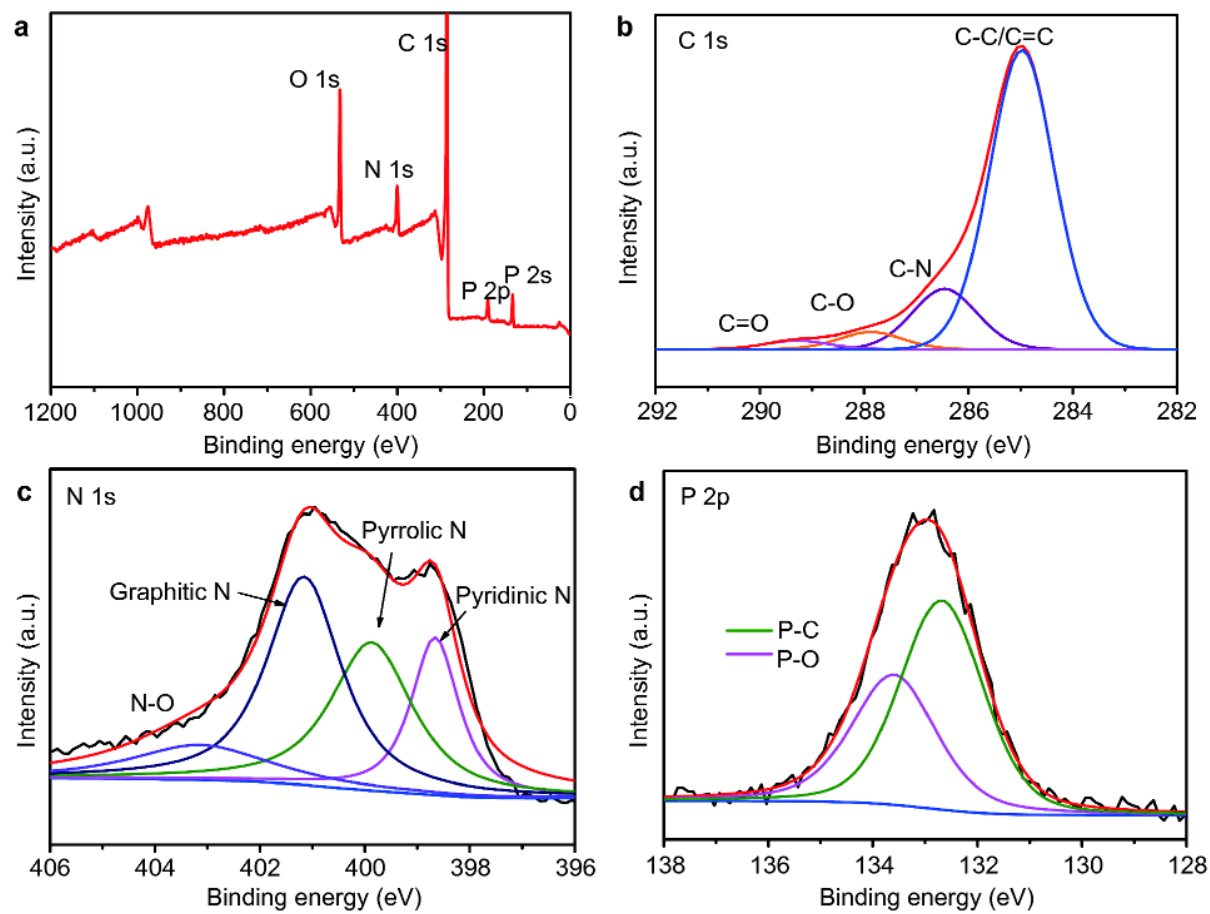

Figure 3 (a) XPS survey spectra of MPSA-Co-900-Acid, (b-d) are the spectra of C 1s, N 1s and P 2p respectively. In C 1s spectrum, the peak at about $284.6 \mathrm{eV}$ was assigned to the graphitic $\mathrm{sp}^{2}$ carbon while the additional component centred at $286.4 \mathrm{eV}$ was attributable to C-N. The $\mathrm{N} 1 \mathrm{~s}$ spectrum is deconvoluted into four $\mathrm{N}$ species: pyridinic $\mathrm{N}(398.6 \mathrm{eV})$, pyrrolic $\mathrm{N}$ (399.9 eV), graphitic $\mathrm{N}(401.1 \mathrm{eV})$, and oxidized N (403.4 eV), respectively. P 2p spectrum had two typical peaks: P-C (132.7 eV) and P-O (133.6 eV)[38].

layers. The high resolution image in Fig. $2 b$ exhibits that the hole size was nearly $20 \mathrm{~nm}$. These holes would expose more extra active edge sites for ORR and bring better performance $[34,35]$. The surface area from the BET experiments also proved that, after the acid washing, the surface area of MPSA-Co-900-Acid $\left(124 \mathrm{~m}^{2} \mathrm{~g}^{-1}\right)$ was a little higher than that of MPSA-Co-900 $\left(102 \mathrm{~m}^{2} \mathrm{~g}^{-1}\right)$, which would bring more active sites.

Raman spectroscopy was an efficient tool to investigate the electronic properties of various carbon materials. The Raman spectra of carbon materials have the typical fea- tures at $1,590 \mathrm{~cm}^{-1}$ (G-band around) and $1,350 \mathrm{~cm}^{-1}(\mathrm{D}$ band around) [36,37]. The intensity ratio of $D$ and $G$ band, $I_{\mathrm{D}} / I_{\mathrm{G}}$, representing the defect level of carbon materials, increased a little after acid washing (Fig. S4). This indicated that the holes formed after acid washing brought much defect sites.

XPS was taken to study the chemical composition and chemical states of different samples (Fig. 3). The survey spectrum of MPSA-Co-900-Acid was shown in Fig. 3a and survey spectra of MPSA-900 and MPSA-Co-900 were provided in Fig. S5a. All the samples have typical peaks of 

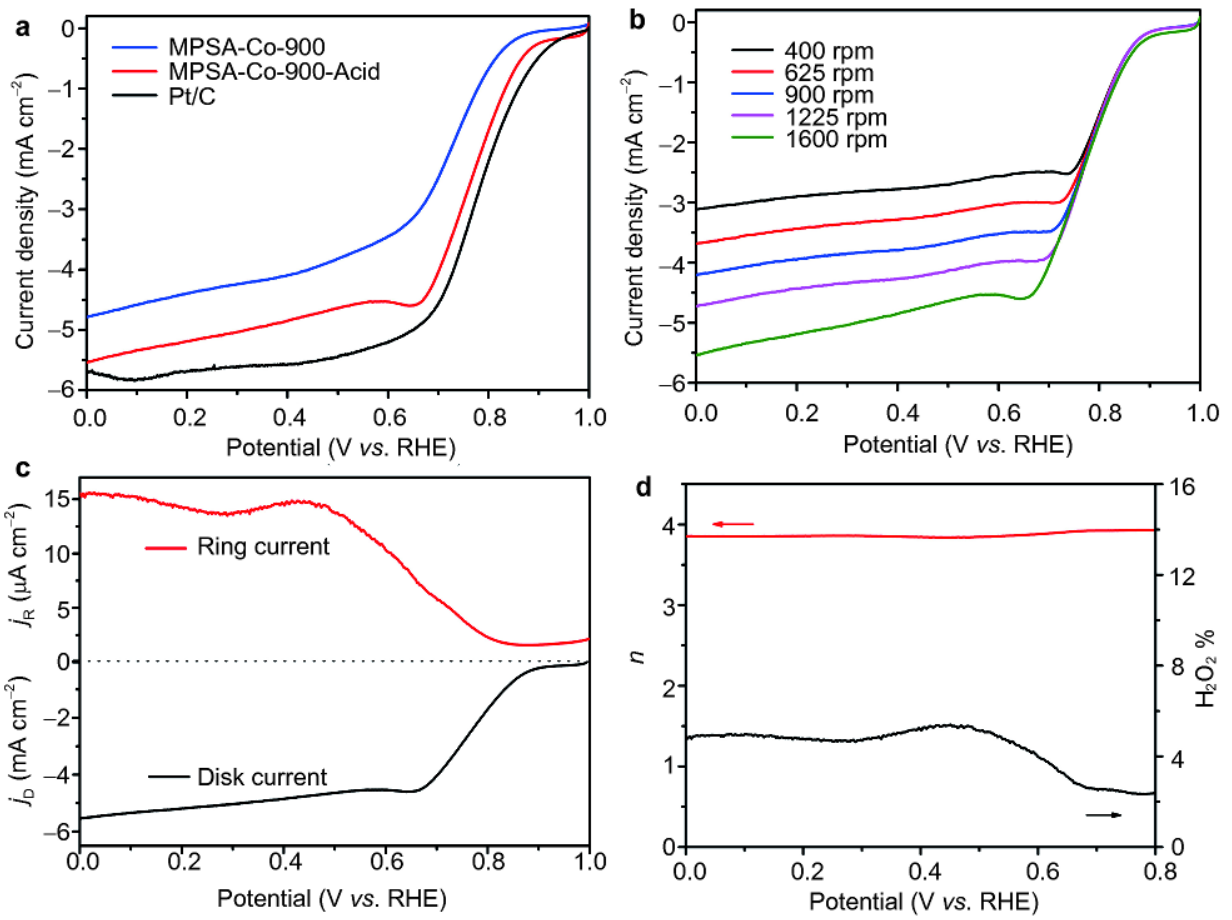

Figure 4 (a) Rotating disk electrode (RDE) voltammograms of MPSA-Co-900, MPSA-Co-900-Acid and Pt/C in an $\mathrm{O}_{2}$-saturated $0.1 \mathrm{~mol} \mathrm{~L} \mathrm{KOH}^{-1} \mathrm{KOH}$ solution with a scan rate of $10 \mathrm{mV} \mathrm{s}^{-1}$. (b) RDE of MPSA-Co-900-Acid at different rotation rates from 400 to $1600 \mathrm{rpm}$. (c) RRDE measurements for MPSA-Co-900-Acid electrode in $\mathrm{O}_{2}$-saturated $0.1 \mathrm{~mol} \mathrm{~L}^{-1} \mathrm{KOH}$ and (d) percentage of peroxide in the total oxygen reduction products together with the number of electron transfer.

C, N, P, O element, indicating the successful doping of N, $\mathrm{P}$ into the carbon nanosheets. In addition, MPSA-Co-900 showed the typical Co peaks. After washed with acid, the sample of MPSA-Co-900-Acid showed nearly no typical peaks of Co, indicating that most of the Co had been removed from the material (Fig. S5b). Only $0.15 \%$ Co in MPSA-Co-900-Acid (Table S1) was, much lower than that of MPSA-Co-900, proved that most of the metal Co has been dissolved, and both the amount of doped $\mathrm{N}$ and $P$ were slightly increased.

The cyclic voltammogram (CV) tests were taken to investigate the electrocatalytic activity of the different carbon catalysts toward ORR in $\mathrm{N}_{2}$ or $\mathrm{O}_{2}$-saturated 0.1 mol L ${ }^{-1} \mathrm{KOH}$ electrolyte at a scan rate of $50 \mathrm{mV} \mathrm{s}^{-1}$ (Fig. S6). The CV curves showed that a quasi-rectangular double-layer capacitance current in $\mathrm{N}_{2}$-saturated electrolyte while an obvious reduction process was found in $\mathrm{O}_{2}$-saturated $0.1 \mathrm{~mol} \mathrm{~L}^{-1} \mathrm{KOH}$ solution. We can see that the sample of MPSA-Co-900-Acid has more positive onset potential and reduction potential than that of MPSA-Co-900, indicating its better ORR performance due to more edge sites exposed after acid washing. To further study the electrocatalytic activity and the kinetics of ORR process on MPSA-900, MPSA-Co-900 and MPSA-Co-900-Acid, linear sweep voltammograms (LSVs) were carried out on a rotating disk electrode $(\mathrm{RDE})$ at rotation rate of $1,600 \mathrm{rpm}$ in $\mathrm{O}_{2}$-saturated 0.1 mol L ${ }^{-1} \mathrm{KOH}$ electrolyte at a scan rate of $10 \mathrm{mV} \mathrm{s}^{-1}$ (Fig. 4a). The curve of MPSA-Co-900-Acid showed obviously more positive onset potential and the half-wave potential than that of MPSA-Co-900 and MPSA-900 (Fig. S7), which was close to that of the commercial $\mathrm{Pt} / \mathrm{C}$. This clearly indicated that both the Co doping and exposing more edge sites can further enhance the activity of the heteroatom doped carbon materials. Even the trace level Co doping can significantly improve the ORR activity of the carbon-based materials. And the activity of MPSACo-900-Acid was better or close to that of other related carbon-based materials shown in Table S2. Moreover, the unique current plateau from 0.7 to $0 \mathrm{~V}$ observed in MPSA-Co-900-Acid showed a typical diffusion-controlled process corresponding to the efficient four electron-controlled ORR pathway. The LSV curves in Fig. 4b showed that the expected current density increased with the increasing scanning rates.

To further quantitatively characterize the sample of 
MPSA-Co-900-Acid, we calculated the transferred electron number $(n)$ per oxygen molecule and the $\mathrm{H}_{2} \mathrm{O}_{2}$ production yield in the oxygen reduction process. Thus, the RRDE test of different samples was provided (Fig. 4c) and the results were calculated according to the formula as follows:

$$
\begin{gathered}
n=4 \frac{I_{\mathrm{d}}}{I_{\mathrm{d}}+I_{\mathrm{r}} / N}, \\
\mathrm{H}_{2} \mathrm{O}_{2} \%=200 \frac{I_{\mathrm{r}} / N}{I_{\mathrm{d}}+I_{\mathrm{r}} / N},
\end{gathered}
$$

where $n$ is the electron transfer number, $\mathrm{H}_{2} \mathrm{O}_{2} \%$ is the production yield during the ORR process, $I_{\mathrm{D}}$ is the absolute value of the disk current, $I_{R}$ is the absolute value of the ring current, and $N$ is the collection efficiency $(0.37$ in this study). And the calculated results (Fig. 4d) exhibited $n$ of MPSA-Co-900-Acid remained from 3.8 to 3.9. This clearly showed that the ORR on MPSA-Co-900-Acid electrode followed an efficient one-step, four-electron pathway over the entire potential range with a low $\mathrm{H}_{2} \mathrm{O}_{2}$ production yield, which was much close to that of $\mathrm{Pt}$ (Fig. S8), indicating the good ORR performance. Compared to MPSA-Co-900, MPSA-Co-900-Acid has larger $n$ and $\mathrm{H}_{2} \mathrm{O}_{2} \%$ than that of MPSA-Co-900 (Fig. S9). This further showed that MPSA-Co-900-Acid had much better ORR performance due to extra exposed active sites after acid washing.

The methanol crossover effect and electrochemical stability were other important parameters for ORR catalysts. The current-time $(I-t)$ chronoamperometric was performed to study the methanol crossover effect of MPSA-Co-900-Acid and Pt/C. As shown in Fig. 5a, when the methanol was added into the solution, the corresponding amperometric response of the MPSA-Co-900Acid is almost unchanged. However, in contrast, the current of commercial $\mathrm{Pt} / \mathrm{C}$ decreases obviously as soon as the adding of methanol. Fig. 5b indicated that the MPSA-Co-900-Acid catalyst had much better methanol tolerance than $\mathrm{Pt} / \mathrm{C}$. The electrochemical stability is also important in consideration of the long-time application. Therefore, the stability test was conducted in $\mathrm{O}_{2}$-saturated $0.1 \mathrm{~mol} \mathrm{~L}^{-1} \mathrm{KOH}$ solution at $0.78 \mathrm{~V}$ and indicated that the MPSA-Co-900-Acid showed very good stability during the long-time test with only $3.4 \%$ current lost after $20,000 \mathrm{~s}$. However, the corresponding current loss of commercial $\mathrm{Pt} / \mathrm{C}$ at the same condition was much higher than that of MPSA-Co-900-Acid, close to $20.2 \%$. Therefore, both the methanol crossover effect and electrochemical stability of MPSA-Co-900-Acid were better than that of $\mathrm{Pt} / \mathrm{C}$.
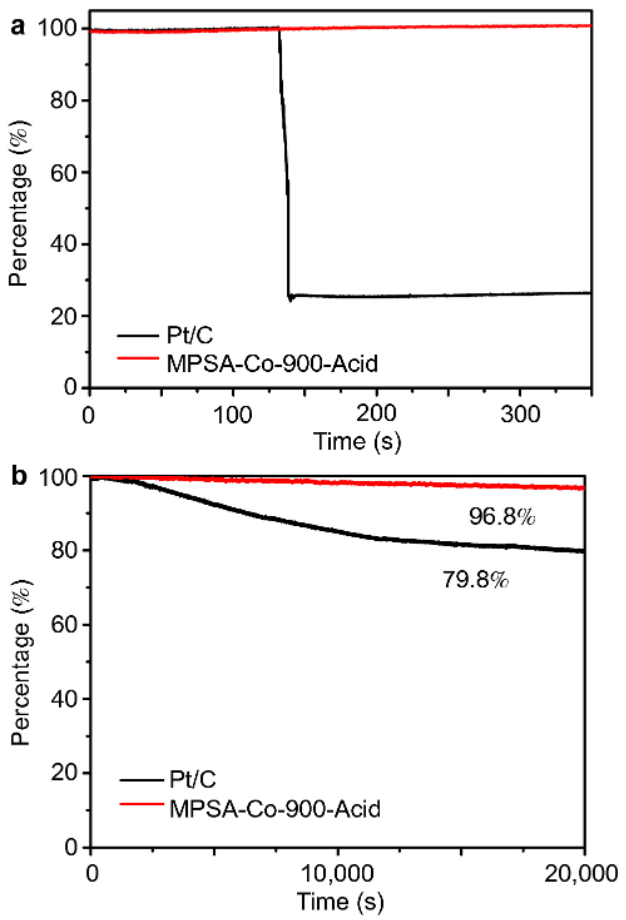

Figure 5 (a) Chronoamperometric responses for MPSA-Co-900-Acid and $\mathrm{Pt} / \mathrm{C}$ electrodes on addition of $1.0 \mathrm{~mol} \mathrm{~L}^{-1}$ methanol after about $150 \mathrm{~s}$ and (b) electrochemical durability tests of MPSA-Co-900-Acid and $\mathrm{Pt} / \mathrm{C}$ electrodes.

\section{CONCLUSIONS}

In summary, we developed an efficient method for the scalable preparion of $\mathrm{N}, \mathrm{P}$ dual-doped carbon material with trace level Co doping by self-assembling melaminephytic acid supermolecular aggregate in the presence of Co, followed by pyrolysis. After washing with acid to remove the metal Co, many holes would be formed on the nanosheets to expose more active sites for ORR. Combining the trace level Co doping and rich edge sites in the dual doped carbon nanosheets, the MPSA-Co-900-Acid showed very good ORR activity close to that of Pt/C. In addition, the electrochemical results showed that MPSACo-900-Acid had much better fuel selectivity and higher long-term stability than that of commercially available Pt/ $\mathrm{C}$ electrode. The strategy developed here could guide researchers to develop ORR electrocatalysts with more active sites exposed.

Received 23 October 2017; accepted 30 November 2017; published online 29 December 2017

1 Dai L, Xue Y, Qu L, et al. Metal-free catalysts for oxygen reduction reaction. Chem Rev, 2015, 115: 4823-4892 
2 Gong K, Du F, Xia Z, et al. Nitrogen-doped carbon nanotube arrays with high electrocatalytic activity for oxygen reduction. Science, 2009, 323: 760-764

3 Jasinski R. A new fuel cell cathode catalyst. Nature, 1964, 201: 1212-1213

4 Wang S, Jiang SP. Prospects of fuel cell technologies. Nat Sci Rev, 2017, 4: 163-166

5 Shao M, Chang Q, Dodelet JP, et al. Recent advances in electrocatalysts for oxygen reduction reaction. Chem Rev, 2016, 116: 3594-3657

6 Wang S, Zhang L, Xia Z, et al. BCN graphene as efficient metalfree electrocatalyst for the oxygen reduction reaction. Angew Chem Int Ed, 2012, 51: 4209-4212

7 Zhao C, Yu C, Liu S, et al. 3D porous N-doped graphene frameworks made of interconnected nanocages for ultrahigh-rate and long-life $\mathrm{Li}_{-} \mathrm{O}_{2}$ batteries. Adv Funct Mater, 2015, 25: 6913-6920

8 Zhou T, Du Y, Yin S, et al. Nitrogen-doped cobalt phosphate@ nanocarbon hybrids for efficient electrocatalytic oxygen reduction. Energy Environ Sci, 2016, 9: 2563-2570

9 Yang L, Jiang S, Zhao Y, et al. Boron-doped carbon nanotubes as metal-free electrocatalysts for the oxygen reduction reaction. Angew Chem Int Ed, 2011, 50: 7132-7135

10 Xia BY, Yan Y, Li N, et al. A metal-organic framework-derived bifunctional oxygen electrocatalyst. Nat Energy, 2016, 1: 15006

11 Wang S, Yu D, Dai L. Polyelectrolyte functionalized carbon nanotubes as efficient metal-free electrocatalysts for oxygen reduction. J Am Chem Soc, 2011, 133: 5182-5185

12 Guo D, Shibuya R, Akiba C, et al. Active sites of nitrogen-doped carbon materials for oxygen reduction reaction clarified using model catalysts. Science, 2016, 351: 361-365

13 Liang HW, Wei W, Wu ZS, et al. Mesoporous metal-nitrogendoped carbon electrocatalysts for highly efficient oxygen reduction reaction. J Am Chem Soc, 2013, 135: 16002-16005

14 Zhang G, Jin X, Li H, et al. N-doped crumpled graphene: bottomup synthesis and its superior oxygen reduction performance. Sci China Mater, 2016, 59: 337-347

$15 \mathrm{Wu} \mathrm{S}$, Zhu Y, Huo Y, et al. Bimetallic organic frameworks derived $\mathrm{CuNi} /$ carbon nanocomposites as efficient electrocatalysts for oxygen reduction reaction. Sci China Mater, 2017, 60: 654-663

16 Wang L, Jia W, Liu X, et al. Sulphur-doped ordered mesoporous carbon with enhanced electrocatalytic activity for the oxygen reduction reaction. J Energy Chem, 2016, 25: 566-570

17 Seredych M, László K, Rodríguez-Castellón E, et al. S-doped carbon aerogels/GO composites as oxygen reduction catalysts. J Energy Chem, 2016, 25: 236-245

18 Preuss K, Kannuchamy VK, Marinovic A, et al. Bio-inspired carbon electro-catalysts for the oxygen reduction reaction. J Energy Chem, 2016, 25: 228-235

19 Zhang J, Dai L. Heteroatom-doped graphitic carbon catalysts for efficient electrocatalysis of oxygen reduction reaction. ACS Catal, 2015, 5: 7244-7253

20 Zhang L, Xia Z. Mechanisms of oxygen reduction reaction on nitrogen-doped graphene for fuel cells. J Phys Chem C, 2011, 115: 11170-11176

21 Xiao Z, Wang $\mathrm{Y}$, Huang $\mathrm{YC}$, et al. Filling the oxygen vacancies in $\mathrm{Co}_{3} \mathrm{O}_{4}$ with phosphorus: an ultra-efficient electrocatalyst for overall water splitting. Energy Environ Sci, 2017, 44

22 Dou S, Dong CL, Hu Z, et al. Atomic-scale $\mathrm{CoO}_{x}$ species in metalorganic frameworks for oxygen evolution reaction. Adv Funct Mater, 2017, 27: 1702546
23 Zhang J, Qu L, Shi G, et al. N,P-codoped carbon networks as efficient metal-free bifunctional catalysts for oxygen reduction and hydrogen evolution reactions. Angew Chem Int Ed, 2016, 55: 2230-2234

24 Chang Y, Hong F, He C, et al. Nitrogen and sulfur dual-doped non-noble catalyst using fluidic acrylonitrile telomer as precursor for efficient oxygen reduction. Adv Mater, 2013, 25: 4794-4799

25 Wang X, Wang J, Wang D, et al. One-pot synthesis of nitrogen and sulfur co-doped graphene as efficient metal-free electrocatalysts for the oxygen reduction reaction. Chem Commun, 2014, 50: 48394842

26 Shen A, Zou Y, Wang Q, et al. Oxygen reduction reaction in a droplet on graphite: direct evidence that the edge is more active than the basal plane. Angew Chem Int Ed, 2014, 53: 10804-10808

27 Tang C, Wang HF, Chen X, et al. Topological defects in metal-free nanocarbon for oxygen electrocatalysis. Adv Mater, 2016, 28: 6845-6851

28 Yan D, Li Y, Huo J, et al. Defect chemistry of nonprecious-metal electrocatalysts for oxygen reactions. Adv Mater, 2017, 414: 1606459

29 Tang C, Wang B, Wang HF, et al. Defect engineering toward atomic $\mathrm{Co}-\mathrm{N}_{x}-\mathrm{C}$ in hierarchical graphene for rechargeable flexible solid Zn-air batteries. Adv Mater, 2017, 29: 1703185

30 Liu Z, Zhao Z, Wang Y, et al. In situ exfoliated, edge-rich, oxygenfunctionalized graphene from carbon fibers for oxygen electrocatalysis. Adv Mater, 2017, 29: 1606207

$31 \mathrm{Wu}$ G, More KL, Johnston CM, et al. High-performance electrocatalysts for oxygen reduction derived from polyaniline, iron, and cobalt. Science, 2011, 332: 443-447

32 Chen Y, Gokhale R, Serov A, et al. Novel highly active and selective Fe-N-C oxygen reduction electrocatalysts derived from in-situ polymerization pyrolysis. Nano Energy, 2017, 38: 201-209

33 Hu K, Xiao Z, Cheng Y, et al. Iron phosphide/N, P-doped carbon nanosheets as highly efficient electrocatalysts for oxygen reduction reaction over the whole $\mathrm{pH}$ range. Electrochim Acta, 2017, 254: 280-286

34 Jiang Y, Yang L, Sun T, et al. Significant contribution of intrinsic carbon defects to oxygen reduction activity. ACS Catal, 2015, 5: 6707-6712

35 Tao L, Wang Q, Dou S, et al. Edge-rich and dopant-free graphene as a highly efficient metal-free electrocatalyst for the oxygen reduction reaction. Chem Commun, 2016, 52: 2764-2767

36 Yan D, Dou S, Tao L, et al. Electropolymerized supermolecule derived N, P co-doped carbon nanofiber networks as a highly efficient metal-free electrocatalyst for the hydrogen evolution reaction. J Mater Chem A, 2016, 4: 13726-13730

37 Sun $\mathrm{M}$, Zhang G, Liu $\mathrm{H}$, et al. $\alpha$ - and $\gamma-\mathrm{Fe}_{2} \mathrm{O}_{3}$ nanoparticle/nitrogen doped carbon nanotube catalysts for high-performance oxygen reduction reaction. Sci China Mater, 2015, 58: 683-692

38 Zhang J, Zhao Z, Xia Z, et al. A metal-free bifunctional electrocatalyst for oxygen reduction and oxygen evolution reactions. Nat Nanotechnol, 2015, 10: 444-452

Acknowledgements This work was supported by the National Natural Science Foundation of China (21701043, 21573066, and 51402100), the Provincial Natural Science Foundation of Hunan (2016JJ1006 and 2016TP1009), the Key Laboratory of Optoelectronic Devices and Systems of Ministry of Education and Guangdong Province and Shenzhen Science and Technology Program (JCYJ20170306141659388). 
Author contributions Yan D and Guo $\mathrm{L}$ designed and engineered the samples; Yan D, Guo L, Xie C, Wang Y, Li Y, Li H performed the experiment and the characterizations. Yan D and Guo L wrote the paper with support from Wang S. All authors contributed to the general discussion.
Conflict of interest The authors declare that they have no conflict of interest.

Supplementary information Supporting data are available in the online version of the paper.

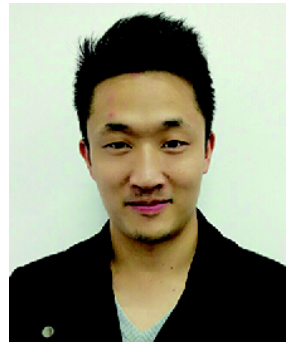

Dafeng Yan received his BSc degree in 2014 from the Department of Chemistry, Hunan University, China. He is currently pursuing his $\mathrm{PhD}$ degree under the supervision of Prof. Shuangyin Wang. His current interests include the synthesis and characterization of nanomaterials with various defects and the relationship between the defects and the electrocatalytic performance of renewable energy-related reactions.

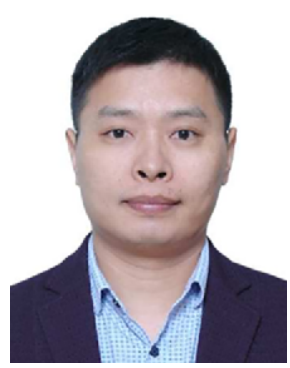

Shuangyin Wang received his BSc degree in 2006 from Zhejiang University and $\mathrm{PhD}$ in 2010 from Nanyang Technological University, Singapore. He was a postdoctoral fellow working with Prof. Liming Dai (2010-2011) and Prof. A. Manthiram (2011-2012). He is currently a Professor of Hunan University. His research interests are in novel carbon catalysts, defects in various crystals and their application on electrocatalysis and batteries.

\section{具有痕量钴掺杂且暴露更多边缘活性的 N,P共掺杂的多孔碳纳米片作为高效氧还原催化剂}

严大峰 ${ }^{1 \dagger}$, 郭兰 ${ }^{1 \dagger}$, 谢超 ${ }^{1}$, 王燕勇 ${ }^{1}$, 李云霄 ${ }^{1}$, 李浩 ${ }^{1}$, 王双印 $卬^{1,2,3^{*}}$

摘要 本文使用有机分子配位聚合作用一步聚合、碳化、酸洗得到了一种 N,P双掺杂碳材料. 其具有痕量掺杂的金属钴、且具有更多活 性边缘. X射线光电子能谱显示杂原子成功进入碳材料当中, 并且发现酸洗后钴的信号非常低, 证明酸洗后, 材料表面形成非常多的孔, 暴 露出更多的边缘催化位点. 制备的碳材料具有大量催化活性位点, 因此表现出极其优异的电催化氧还原性能. 另外, 与 $\mathrm{Pt} / \mathrm{C}$ 相比, 制备的多 孔碳材料还具有较好的抗毒性与稳定性, 进一步显示了其在新能源电池领域的应用前景. 